\title{
Preoperative ferritin and hemoglobin levels are lower in patients with a history of COVID-19 but blood loss and transfusion requirements are not increased
}

\author{
Anna Jungwirth-Weinberger ${ }^{1,2}$. llya Bendich ${ }^{1}$. Geoffrey H. Westrich ${ }^{1} \cdot$ Edwin P. Su ${ }^{1}$. \\ Alejandro Gonzalez Della Valle ${ }^{1} \cdot$ Friedrich Boettner $^{1}[$
}

Received: 16 April 2021 / Accepted: 18 July 2021 / Published online: 24 July 2021

(c) The Author(s), under exclusive licence to Springer-Verlag GmbH Germany, part of Springer Nature 2021

\begin{abstract}
Introduction A history of COVID-19 (Coronavirus Disease 2019), caused by severe acute respiratory syndrome coronavirus 2 (SARS-CoV-2) may have an impact on hemoglobin and ferritin levels predisposing patients to increased blood transfusion requirements following total joint arthroplasty (TJA). The current study compares ferritin levels, hemoglobin levels, and transfusion rates between SARS-CoV-2 IgG positive and SARS-CoV-2 IgG negative TJA patients.

Materials and methods Preoperative ferritin levels, pre- and postoperative hemoglobin levels, postoperative change in hemoglobin, and transfusion rates of 385 consecutive SARS-CoV-2 IgG positive patients undergoing TJA were compared to those of 5156 consecutive SARS-CoV-2 IgG negative patients undergoing TJA.

Results Preoperative hemoglobin levels were significantly lower in SARS-CoV-2 IgG positive patients [13.3 g/dL (range $8.9-17.7 \mathrm{~g} / \mathrm{dL})$ ] compared to $13.5 \mathrm{~g} / \mathrm{dl}(7.3-18.3 \mathrm{~g} / \mathrm{dL} ; p$ value 0.03$)$. Ferritin levels were significantly lower in SARS-CoV-2 IgG positive patients (mean of $106.1 \mathrm{ng} / \mathrm{ml}(2.1-871.3 .3 \mathrm{ng} / \mathrm{ml}) \mathrm{vs.} 123.7 \mathrm{ng} / \mathrm{ml}(1.4-1985 \mathrm{ng} / \mathrm{ml})$ ( $p$ value 0.02$)$ ). Hemoglobin on postoperative day (POD) one, after four-six weeks, and transfusion rates did not differ between the two groups. Conclusion Although preoperative hemoglobin and ferritin levels are lower in SARS-CoV2 IgG positive patients, there was no difference in hemoglobin on POD one, recovery of hemoglobin levels at four-six weeks postoperatively, and transfusion rates after surgery. Routine ferritin testing prior to TJA is not recommended in SARS-CoV-2 IgG positive patients.
\end{abstract}

Keywords COVID-19 $\cdot$ SARS-CoV-2 IgG positive $\cdot$ Ferritin $\cdot$ Hemoglobin $\cdot$ Transfusion

\section{Introduction}

The novel Coronavirus (SARS-CoV-2) pandemic has placed an immense strain on health care systems across the world. With the return of elective surgery, such as TJA, surgeons are now treating an increasing number of patients who have recovered from COVID-19 (Coronavirus Disease 2019). It is important to understand how these patients compare to those unaffected by COVID-19 with regards to preoperative optimization and postoperative recovery.

Friedrich Boettner

boettnerf@hss.edu

1 Hospital for Special Surgery, 535 East 70th Street, New York, NY 10021, USA

2 Cantonal Hospital Baden, Im Ergel 1, CH-5404 Baden, Switzerland
Under normal circumstances, serum ferritin levels are a sensitive marker for iron status. Low blood levels of ferritin may be present in cases of iron deficiency anemia [10]. However, ferritin is also an acute-phase reactant that becomes elevated in response to acute inflammation [5], as proinflammatory cytokines trigger an increase in hepcidin, which in turn restricts uptake of dietary iron and promotes sequestration of iron by ferritin within storage sites [12]. Patients with inflammatory conditions may thus have restricted availability of iron for erythropoiesis and other cell functions due to increased hepcidin expression, despite normal or high levels of serum ferritin [2].

Ferritin has been shown to be elevated during the acute phase of SARS (Severe Acute Respiratory Syndrome), MERS (Middle East Respiratory Syndrome), COVID-19, infectious as well as non-infectious diseases [8]. Elevated Ferritin blood levels during the acute phase of COVID19 represent a poor prognostic marker $[15,16,18]$ and an 
independent risk factor for the development of Acute Respiratory Distress Syndrome (ARDS) [4]. Higher levels of ferritin also correlate with more severe COVID-19 cases [9].

Hemoglobin levels in patients infected with SARS-CoV-2 are lower [14] than that of the general population. Among COVID-19 patients requiring intensive care unit (ICU) level care, hemoglobin levels are significantly lower than those not requiring ICU level care [1].

Iron deficiency is the most common cause for anemia in patients undergoing total joint arthroplasty (TJA) [7]. Preoperative anemia is associated with increased postoperative transfusion requirements [3]. Oral iron therapy increased hemoglobin and serum ferritin levels in a cohort of 3,435 TJAs and reduced utilization of other blood-conserving therapies before total hip arthroplasty (THA) and total knee arthroplasty (TKA) [11].

The current study aims to compare preoperative hemoglobin and ferritin levels, intraoperative blood loss, transfusion rates, and postoperative recovery of hemoglobin levels in SARS-CoV-2 IgG positive patients and negative patients undergoing elective TJA.

\section{Materials and methods}

The study was approved by the local ethics review board and Institutional Review Board (IRB).

Hemoglobin and ferritin levels were collected in a consecutive series of patients undergoing TJA between May 2020 and January 2021 at the author's institution.

Patients undergoing elective primary TJA including THA and TKA were included in the current study. Patients with missing preoperative hemoglobin levels, revision surgeries, and simultaneous bilateral surgeries were excluded.

The inclusion criteria were met by 5,156 SARS-CoV-2 IgG negative and 385 SARS-CoV-2 IgG positive patients. The polymerase chain reaction (PCR) test for active SARSCoV-2 infection was negative in all patients at the time of surgery. SARS-CoV-2 IgG positive patients, who recovered from COVID-19 and subsequently tested negative on PCR test prior to surgery underwent TJA.

Hemoglobin and ferritin levels were drawn during presurgical screening one to 21 days prior to surgery.

Postoperative hemoglobin values and the number of patients needing a blood transfusion were evaluated. In addition, 368 patients (255 SARS-CoV-2 IgG negative and 113 SARS-CoV-2 IgG positive) had follow-up hemoglobin four-six weeks after surgery.

Hemoglobin levels were determined preoperatively in 5,156 SARS-CoV-2 IgG negative and 385 SARSCoV-2 IgG positive patients. Normal Hemoglobin ranges were categorized as follows: $13.3-17.2 \mathrm{~g} / \mathrm{dL}$ in men and $12.0-15.0 \mathrm{~g} / \mathrm{dL}$ in women, as per the institution's normal range. We recorded hemoglobin levels determined preoperatively, on POD one and four to six weeks postoperatively. We calculated hemoglobin drop subtracting hemoglobin on POD one from preoperative, and hemoglobin increase by subtracting hemoglobin on POD one from hemoglobin after four-six weeks.

Transfusion policy at our institution is to transfuse for hemoglobin levels below $8 \mathrm{~g} / \mathrm{dl}$ in patients with clinical symptoms of anemia (tachycardia, hypotension) that did not response to a fluid bolus.

Ferritin levels were collected preoperatively in 2158 SARS-CoV-2 IgG negative and 264 SARS-CoV-2 IgG positive patients. Normal ferritin values in men range between 22 and $275 \mathrm{ng} / \mathrm{ml}$ and in women between 5 and $204 \mathrm{ng} / \mathrm{ml}$, as per the institution's normal range.

Tranexamic acid was routinely used in all patients, either topical ( $3 \mathrm{~g})$, intravenous ( $1 \mathrm{~g}$ ) or two intravenous doses $(1 \mathrm{~g})$.

The SARS-CoV-2 IgG positive group consisted of 210 female and 175 male patients, the SARS-CoV-2 IgG negative group of 3,074 female and 2082 male patients. Demographic information is presented in Table 1.

191 SARS-CoV-2 IgG positive patients underwent a THA and 194 patients a TKA. The SARS-CoV-2 IgG negative group consisted of 2800 THAs and 2356 TKAs.

Analyses were conducted using SAS software version 9.4 (SAS Institute Inc., Carey, NC). Mean with range, as well as $95 \%$ confidence interval, or median with interquartile range were presented accordingly. Continuous variables were evaluated using Wilcoxon signed-rank test. Categorical variables were analyzed using chi-squared tests, or where appropriate Fisher's exact test. $p$ values $<0.05$ were considered significantly different.
Table 1 Demographic information of the two study groups

\begin{tabular}{lllc}
\hline & SARS-CoV-2 IgG positive & SARS-CoV-2 IgG negative & $p$ value \\
\hline$n$ & 385 & 5156 & \\
Age (years) & $63.7(16-92)$ & $65.5(15-96)$ & 0.001 \\
Male/female & $175 / 210$ & $2082 / 3074$ & 0.05 \\
BMI $\left(\mathrm{kg} / \mathrm{m}^{2}\right)$ & $31.1(16.8-52.3)$ & $29.6(14.6-58.6)$ & $<0.0001$ \\
\hline
\end{tabular}




\section{Results}

The average ferritin was $106.1 \mathrm{ng} / \mathrm{ml}(2.1-871.3 \mathrm{ng} / \mathrm{ml})$ in SARS-CoV-2 IgG positive TJA patients compared to $123.7 \mathrm{ng} / \mathrm{ml}(1.4-1985 \mathrm{ng} / \mathrm{ml})$ in SARS-CoV-2 IgG negative TJA patients (Fig. 1) $(p=0.02)$.

In the SARS-CoV-2 IgG negative group, 253 patients (4.9\%) had abnormal ferritin levels. Among this group, 221 patients $(87.4 \%$; 116 male/105 female; mean age 66 years; mean BMI $30.4 \mathrm{~kg} / \mathrm{m}^{2}$ ) had elevated ferritin levels and 32 patients $(12.6 \%$; 26 male/6 female; mean age 66 years; mean BMI $30.5 \mathrm{~kg} / \mathrm{m}^{2}$ ) had decreased ferritin levels.

In the SARS-CoV-2 IgG positive group, 28 patients (7.3\%) had abnormal ferritin levels. Among this group, 18 patients $(64.3 \%$; 11 male/7 female; average age 62 years, average BMI $29.1 \mathrm{~kg} / \mathrm{m}^{2}$ ) had elevated ferritin levels and 10 patients ( $35.7 \%$; 8 male/ 2 female; average age 63 years; average BMI $31.9 \mathrm{~kg} / \mathrm{m}^{2}$ ) decreased levels. Significantly more patients in the SARS-CoV-2 IgG positive group had abnormal ferritin levels than in the SARS-CoV-2 IgG negative group $(p<0.04)$.

The mean preoperative hemoglobin levels were $13.0 \mathrm{~g} /$ dL (range 7.9-16.8 g/dL) in SARS-CoV-2 IgG positive patients and $13.5 \mathrm{~g} / \mathrm{dL}(7.3-18.3 \mathrm{~g} / \mathrm{dL})$ in SARS-CoV-IgG negative patients. Preoperative hemoglobin values were significantly lower in the SARS-CoV-2 IgG positive group $(p=0.03)$.

The mean hemoglobin level on POD one in the SARS$\mathrm{CoV}-2 \mathrm{IgG}$ positive group was $11.4 \mathrm{~g} / \mathrm{dL}$ (7.5-15.6 g/ $\mathrm{dL})$ compared to $11.5 \mathrm{~g} / \mathrm{dL}(4.1-16.6 \mathrm{~g} / \mathrm{dL})$ in the SARSCoV-2 IgG negative group (Fig. 2). Hemoglobin levels on POD one did not differ significantly between the two groups $(p=0.6)$, and the decrease in hemoglobin from pre- to postoperative was also not significantly different $(p<0.8)$ with a decrease of $-1.9 \mathrm{~g} / \mathrm{dL}(-4.8-2.0 \mathrm{~g} /$ $\mathrm{dL}$ ) in the SARS-CoV-2 IgG positive group and $-1.9 / \mathrm{dL}$ $(-4.8-2.0 \mathrm{~g} / \mathrm{dL})$ in the SARS-CoV-2 IgG negative group, respectively.

Hemoglobin levels after four-six weeks increased to $12.3 \mathrm{~g} / \mathrm{dl}(8.9-15.2 \mathrm{~g} / \mathrm{dl})$ in SARS-CoV-2 $\mathrm{IgG}$ positive patients and to $12.1 \mathrm{~g} / \mathrm{dl}(6.7-6.2 \mathrm{~g} / \mathrm{dl})$ in SARS-CoV-2 IgG negative patients $(p=0.3)$. The hemoglobin increase from POD one to four-six weeks postoperatively was not significantly different between the two groups $[0.7 \mathrm{~g} / \mathrm{dL}(-2.4-3.3 /$ $\mathrm{dL})$ versus $0.6 \mathrm{~g} / \mathrm{dL}(-4.1-3.9 \mathrm{~g} / \mathrm{dL})(p=0.2)]$.

Seven patients $(1.8 \%)$ in the SARS-CoV-2 IgG positive group and 111 patients $(2.2 \%)$ in the SARS-CoV-2 IgG negative group required blood transfusions $(p=0.7)$.

None of the patients with abnormal ferritin levels in the SARS-CoV-2 IgG positive group required a blood transfusion; and 6/221 SARS-CoV-2 IgG (2.7\%) negative patients with elevated ferritin levels and $3 / 32(9.4 \%)$ patients with decreased ferritin levels required a blood transfusion $(p=0.3)$.

\section{Discussion}

In this study, we found that preoperative Hemoglobin levels and ferritin levels were lower in TJA patients who had a history of a COVID-19 infection (SARS-CoV-2 IgG positive) compared to those without evidence of the infection (SARS-CoV-2 IgG negative). However, this did not have an impact on the overall drop in hemoglobin levels and transfusion requirements after elective TJA. In addition, although ferritin levels were significantly different between the two groups, there was no evidence that SARS-CoV-2 IgG
Fig. 1 Preoperative Ferritin levels in SARS-CoV-IgG positive and negative patients

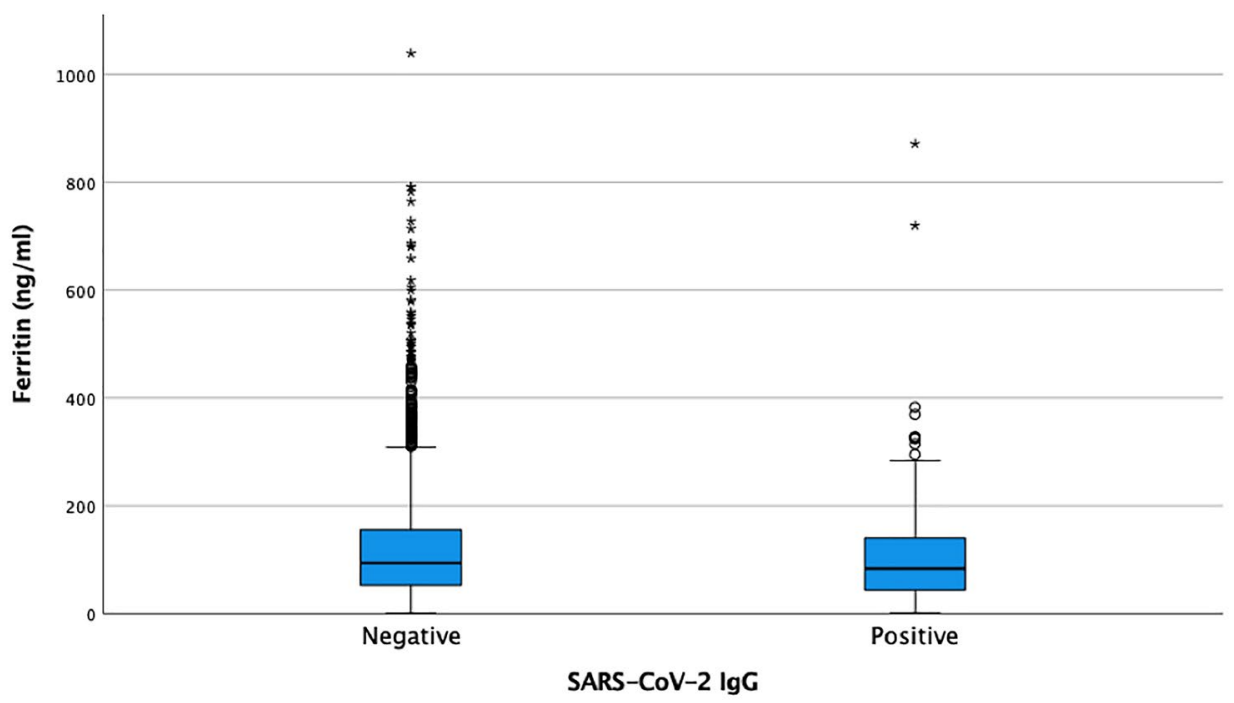


Fig. 2 Hemoglobin levels preoperative and on POD one in SARS-CoV-2 IgG positive and negative patients

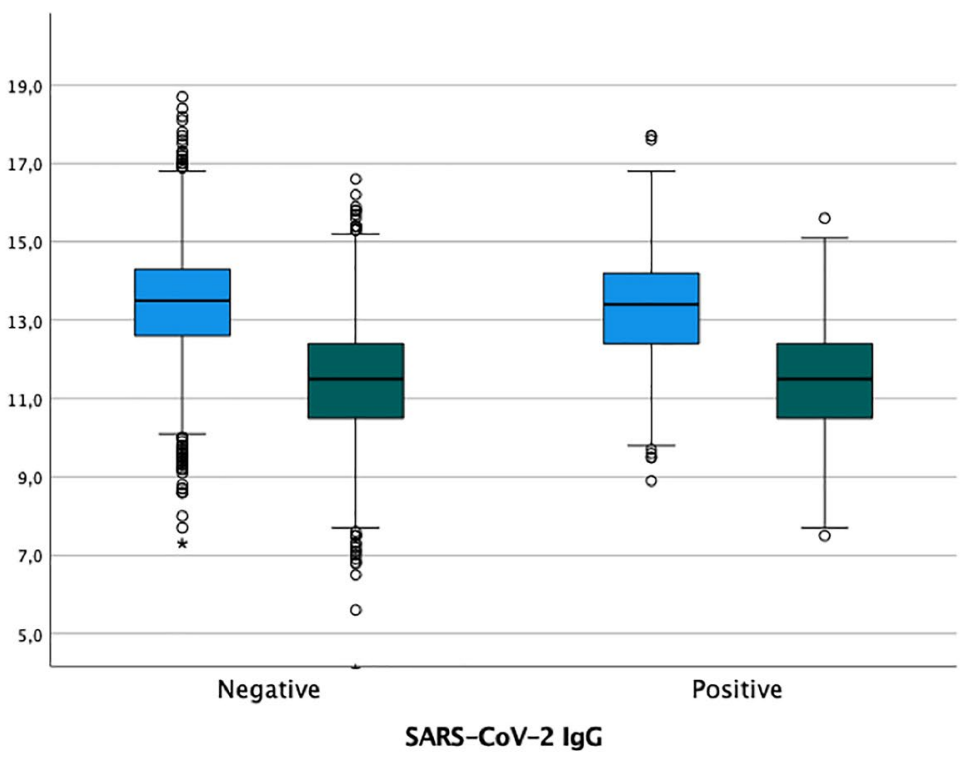

b preoperative $(\mathrm{g} / \mathrm{dl})$ Hb POD1 (g/dl) positive patients recovered hemoglobin levels more slowly within the first four-six weeks after surgery.

The prevalence of iron deficiency in a TJA surgery population was $6 \%$ in a study by Waters et al. [17], but increased to $37.5 \%$ in patients with anemia. Preoperative anemia is strongly associated with increased transfusion requirement [3]. The blood transfusion rate in the current study is $2.2 \%$ among SARS-CoV-2 IgG negative TJA patients and $1.8 \%$ among SARS-CoV-2 IgG positive TJA patients. This rate is lower than what is reported in the literature for nonCOVID-19 infected patients. The use of TXA has dramatically decreased the blood loss and postoperative blood transfusion rate after TJA. Huang et al. reported a postoperative blood transfusion rate of $18.9 \%$ in a large dataset of patients undergoing THA and TKA; the use of TXA decreased the risk of perioperative allogenic blood transfusion [6]. A different study found that 1,534 patients undergoing TJA had a transfusion rate of $17.9 \%$ (7.9\% intraoperatively, and $11.3 \%$ postoperatively, respectively) without the use of TXA [13].

The finding in our study suggests that patients with a history of COVID-19 are not at increased risk of requiring a blood transfusion, and that standard institutional protocols for hemostasis and blood management are appropriate for SARS-CoV-2 IgG positive patients undergoing elective TJA.

Another key finding of this study is that while preoperative ferritin levels differed between for SARS-CoV-2 IgG positive and negative TJA patients, this did not influence transfusion rates or recovery of hemoglobin at four to six weeks postoperatively. Hemoglobin levels between SARS-CoV-2 IgG positive and negative TJA patients at four-six weeks postoperatively were similar. This finding indicates that there is no added benefit of routinely checking ferritin levels among patients with a history of
COVID-19 infection, who are no longer actively infected and scheduled to undergo elective surgery such as TJA.

There are several limitations to this study. (1) The follow-up period is relatively short, although we believe the levels checked are most relevant to the short-term postoperative period; (2) the SARS-CoV-2 IgG positive group consisted of only 385 patients and these patients were not matched to the larger SARS-CoV-2 IgG negative group; (3) the severity of the initial COVID-19 infection was not evaluated for the SARS-CoV-2 IgG positive patients and (4) patients with prolonged course and more severe symptoms might present with more significant anemia and lower ferritin levels. (5) The current study focuses on an early period following the initial outbreak of COVID-19 in New York, suggesting a less than 6 months interval between COVID-19 infection and TJA surgery. However, data on the time interval between surgery and COVID-19 infection were not available and its impact on ferritin or hemoglobin levels remains unclear.

\section{Conclusion}

Although both preoperative hemoglobin and ferritin levels preoperatively were lower in SARS-CoV-2 arthroplasty patients, there was no difference in postoperative hemoglobin levels, blood transfusion rates, and recovery of hemoglobin levels at four-six weeks after surgery. Based upon this study, we do not recommend routine ferritin testing in SARS-CoV-2 IgG positive patients prior to TJA surgery. 
Acknowledgements The authors thank Rachelle Hornick, MPH, for her help with the statistics and Michael Alexiades MD, Michael Ast MD, Jason Blevins MD, Mathias Bostrom MD, Robert Buly MD, Alberto Carli MD, Michael Cross MD, Fred Cushner MD, Mark Figgie MD, Steven Haas MD, Allen Inglis MD, Seth Jerabek MD, David Mayman MD, Douglas Padgett MD, Michael Parks MD, Jose Rodriguez MD, Eduardo Salvati MD, Peter Sculco MD, Thomas Sculco MD, Jonathan Vigdorchik MD, and Russell Windsor MD from the ARJR service for contributing patients to the current study.

Author contributions All authors contributed to the study conception and design. Material preparation, data collection and analysis were performed by AJ-W. The first draft of the manuscript was written by AJ-W and all authors commented on previous versions of the manuscript. All authors read and approved the final manuscript.

Funding No funding was received to assist with the preparation of this manuscript.

\section{Declarations}

Conflict of interest FB receives royalties from Orthodevelopment and Smith and Nephew and compensation from Orthodevelopment, Smith and Nephew, Depuy and Medtronic, unrelated to this research. AGDV receives royalties from Orthodevelopment and Orthosensor and compensation from Johnson and Johnson, unrelated to this research. EPS receives royalties from Smith and Nephew, United Orthopedics and Orthalign, compensation from Smith and Nephew and United Orthopedics, stock or stock options from Orthalign and Engage Surgical, research support from Smith and Nephew and United Orthopedics, and is member of the editorial board of Techniques in Orthopedics and Board member of the AAOS Hip program subcommittee and exhibits committee, unrelated to this research.

Ethical approval The study was approved by the institutional review board.

\section{References}

1. Algassim AA, Elghazaly AA, Alnahdi AS et al (2020) Prognostic significance of hemoglobin level and autoimmune hemolytic anemia in SARS-CoV-2 infection. Ann Hematol 1:1-7

2. Dignass A, Farrag K, Stein J (2018) Limitations of serum ferritin in diagnosing iron deficiency in inflammatory conditions. Int $\mathbf{J}$ Chronic Dis 2018:9394060

3. Frew N, Alexander D, Hood J, Acornley A (2016) Impact of a blood management protocol on transfusion rates and outcomes following total hip and knee arthroplasty. Ann R Coll Surg Engl 98(6):380-386
4. Gandini O, Criniti A, Ballesio L et al (2020) Serum Ferritin is an independent risk factor for acute respiratory distress syndrome in COVID-19. J Infect 81(6):979-997

5. Goldhaber G, Segal G, Dagan A (2020) Hyperferritinemia in the elderly can differentiate the bad from the worst: a retrospective cohort analysis. Medicine (Baltimore) 99(31):e21419

6. Huang Z, Huang C, Xie J et al (2018) Analysis of a large data set to identify predictors of blood transfusion in primary total hip and knee arthroplasty. Transfusion 58(8):1855-1862

7. Jans $\varnothing$, Nielsen CS, Khan N, Gromov K, Troelsen A, Husted H (2018) Iron deficiency and preoperative anaemia in patients scheduled for elective hip- and knee arthroplasty - an observational study. Vox Sang 113(3):260-267

8. Kappert K, Jahić A, Tauber R (2020) Assessment of serum ferritin as a biomarker in COVID-19: bystander or participant? Insights by comparison with other infectious and non-infectious diseases. Biomarkers 25(8):616-625

9. Keske Ş, Tekin S, Sait B et al (2020) Appropriate use of tocilizumab in COVID-19 infection. Int J Infect Dis 99:338-343

10. Moore C Jr, Ormseth M, Fuchs H (2013) Causes and significance of markedly elevated serum ferritin levels in an academic medical center. J Clin Rheumatol 19(6):324-328

11. Petis SM, Lanting BA, Vasarhelyi EM, Naudie DDR, Ralley FE, Howard JL (2017) Is there a role for preoperative iron supplementation in patients preparing for a total hip or total knee arthroplasty? J Arthroplasty 32(9):2688-2693

12 Restrepo-Gallego M, Díaz LE, Rondó PHC (2020) Classic and emergent indicators for the assessment of human iron status. Crit Rev Food Sci Nutr 7:1-14

13. Song K, Pan P, Yao Y, Jiang T, Jiang Q (2019) The incidence and risk factors for allogenic blood transfusion in total knee and hip arthroplasty. J Orthop Surg Res 14(1):273

14. Sun S, Cai X, Wang H et al (2020) Abnormalities of peripheral blood system in patients with COVID-19 in Wenzhou, China. Clin Chim Acta 507:174-180

15. Terpos E, Ntanasis-Stathopoulos I, Elalamy I et al (2020) Hematological findings and complications of COVID-19. Am J Hematol 95(7):834-847

16. Wang F, Hou H, Luo Y, et al. (2020) The laboratory tests and host immunity of COVID-19 patients with different severity of illness. JCI Insight 5(10). https://doi.org/10.1172/jci.insight.137799

17. Waters JH, Johnson P, Yazer MH (2018) Prevalence of iron deficiency in a total joint surgery population. Hematology 23(8):537-541

18. Zhou C, Chen Y, Ji Y, He X, Xue D (2020) Increased serum levels of hepcidin and ferritin are associated with severity of COVID-19. Med Sci Monit 26:e926178

Publisher's Note Springer Nature remains neutral with regard to jurisdictional claims in published maps and institutional affiliations. 